\title{
Electrochemical Microelectrodes Modified by Self-Assembled Stacked Graphene Nanofibers
}

\author{
Yuhua Yu, Xiaosen Chai, Chun Xu and Jia Zhou* \\ ASIC and System State Key Lab, Department of Microelectronics, Fudan University, \\ 220 Handan RD, Shanghai 200433, China
}

(Received October 19, 2011; accepted March 5, 2012)

Key words: electrochemical detection, SGNF, SAM

In this study, we construct stacked graphene platelet nanofibers (SGNFs)/selfassembled monolayer (SAM)/Au electrodes for electrochemical detection by the controllable adsorption of SGNFs onto a SAM of $n$-octadecyl mercaptan $\left(\mathrm{C}_{18} \mathrm{H}_{37} \mathrm{SH}\right)$ at $\mathrm{Au}$ electrodes. The resulting $\mathrm{SGNF} / \mathrm{SAM} / \mathrm{Au}$ electrode demonstrates superior electrochemical properties over both bare $\mathrm{Au}$ and graphite/SAM/Au electrodes. The results of Raman spectroscopy and scanning electron microscopy (SEM) images show that SGNFs have a higher edge-plane density than that of graphite. Cyclic voltammetry (CV) curves indicate that the SGNF/SAM/Au electrode has a higher peak current than the $\mathrm{Au}$ electrode and graphite/SAM/Au electrode. For the SGNF/SAM/Au electrode, the relationship between its peak current and the square root of the scan rate is linear with a fitting slope of 10.638 and correlation coefficient of 0.9968 . It shows an excellent characteristic of Faradic current and thus a high signal-to-noise ratio (SNR) in electrochemical detection. The big peak current and high SNR provide a potentially high sensitivity for electrochemical sensors.

\section{Introduction}

Graphene, a 2D monolayer of $\mathrm{sp}^{2}$-bonded carbon atoms, has attracted much attention regarding fundamental studies and practical aspects because of its distinctive electrical conductivity and novel physicochemical characteristics since it was first experimentally discovered in 2004.(1) Indeed, not only do one-layer graphene materials have interesting properties, multilayer graphene nanostructures are equally interesting and worthy of investigation. When the dimension of the z-axis is greater than the dimension of the $x-y$ axes, the multilayer graphene nanoribbons are called stacked graphene platelet nanofibers (SGNFs, also called platelet graphite nanofibers). ${ }^{(2)}$ Such nanofibers possess

*Corresponding author: e-mail: jia.zhou@fudan.edu.cn 
an exceptionally high ratio of exposed graphene edge sites to basal plane sites. Since they exhibit a surface comprising mainly of electroactive edges, with only one inactive basal plane at each end of the nanofiber, ${ }^{(3)}$ previous researchers have demonstrated that SGNFs show a faster observed heterogeneous electron transfer rate than graphite and carbon nanotubes (CNTs) and superior electrochemical performance when compared to graphite or CNTs. ${ }^{(4,5)}$ It should be noted that such nanofibers are actually complete opposites of CNTs. Therefore, graphene-based nanomaterials (such as SGNFs) are suitable modification materials or dopants of composites on bare electrodes to improve the electrochemical response.

Research on self-assembled monolayers (SAMs) has made considerable progress in the past 30 years, and they are playing an increasingly important role in electrochemistry. Thiols and related molecules have attracted strong interest in SAM study because they can be employed as insulating barriers between an electrode and a redox couple to study long-range electron transfer (ET). ${ }^{(6)}$ However, if nanomaterials are adsorbed on the surface of the molecular layer, ET can be reestablished and even enhanced. (7) Oxidation and reduction can occur by the penetration of electrons through a thiol SAM film. Metal nanoparticles ${ }^{(8)}$ and carbon nanomaterials ${ }^{(9,10)}$ have been used to modify SAMcovered electrodes as sensing elements. Accordingly, taking the advantages of the strong hydrophobic properties and $\pi$-bonding, SGNFs can be adsorbed strongly on a SAM/Au electrode surface to realize the stable modification of the Au electrode.

In this paper, we propose an effective method of constructing SGNF/SAM/Au electrodes. The constructed electrodes are characterized. Such electrodes show a potentially high sensitivity for electrochemical detection.

\section{Materials and Methods}

\subsection{Reagents and equipment}

$n$-Octadecyl mercaptan $\left(\mathrm{C}_{18} \mathrm{H}_{37} \mathrm{SH}\right)$ used in the SAMs was purchased from Flukua. Ferrocenemethanol (FcM) as the redox probe was from Aldrich. $N, N$-Dimethylformamide (DMF) and other reagents were products of Beijing Chemical Corporation, China. Phosphate-buffered saline (0.10 M PBS, $\mathrm{pH}=7.4)$ solution used as an electrolyte was the product of GIBCO (USA). SGNFs were acquired from STREM Chemicals, USA. Deionized (DI) water with a resistance of $18 \mathrm{M} \Omega$ used in solutions was homemade. All the reagents used in the fabrication were of analytical reagent grade.

Electrodes were fabricated on a 3" (100) p-Si substrate with a resistivity of $8 \Omega \cdot \mathrm{cm}$. A piece of platinum filament and a piece of silver filament were prepared to be the counter electrode and reference electrode, respectively.

To fabricate the Au electrode, a physical-vapor-deposition (PVD) sputtering machine (ULVAC, Japan) was utilized. A photolithographic tool was used for electrode patterning (G33, China). To measure the electrochemical response, cyclic voltammetry (CV) was carried out using a CHI660C or CHI1030 electrochemistry workstation $(\mathrm{CH}$ Instruments Inc, USA).

\subsection{Fabrication of $S G N F / S A M / A$ u electrode}

A thick insulating $\mathrm{SiO}_{2}$ layer $(6000 \AA)$ was deposited by thermal oxidation to cover the silicon substrate. Disclike $\mathrm{Au}$ electrodes were designed with a radius of $500 \mu \mathrm{m}$, 
which were the substrate for the SAM. Au/Ti electrodes were patterned and deposited by lithography and PVD. The thickness of $\mathrm{Au} / \mathrm{Ti}$ was about $100 \mathrm{~nm}$. The Ti layer was used to enhance the $\mathrm{Au}$ adhesion to $\mathrm{SiO}_{2}$. The surface crystal orientation of the Au electrode was characterized by X-ray diffraction (XRD) as shown in Fig. 1. It is clear that the electrode is in the $\langle 100\rangle$ direction.

A three-electrode electrochemical system was built, in which the gold discs acted as working electrodes, and an extrinsic platinum filament and silver filament acted as the counter electrode and the reference electrode, respectively. A system based on a similar working mechanism can perform as an electrochemical sensor.

All the solutions were purged with pure nitrogen for at least $15 \mathrm{~min}$ to remove oxygen prior to the experiments. A clean $\mathrm{Au}$ electrode was immersed in an ethanol solution containing $10 \mathrm{mM} \mathrm{C}_{18} \mathrm{H}_{37} \mathrm{SH}$ for at least $48 \mathrm{~h}$ at room temperature $\left(22 \pm 2^{\circ} \mathrm{C}\right)$ to form a $\mathrm{SAM}$ on its surface. The SAM-modified Au electrode (denoted as SAM/Au electrode) was then rinsed with ethanol and dried under pure $\mathrm{N}_{2}$ flow. SGNFs were adsorbed onto the SAM/Au electrode by immersion in $4 \mathrm{mg} / \mathrm{ml} \mathrm{SGNF}$ suspension in DMF for $30 \mathrm{~min}$ at room temperature. The SGNF/SAM/Au electrode was thoroughly rinsed with DMF and DI water, and dried using pure $\mathrm{N}_{2}$ flow before use.

For comparison, flakes of graphite were also used to construct a graphite/SAM/Au electrode. Graphite was absorbed on a SAM using the same steps as those used to make the SGNF/SAM/Au electrode.

\subsection{Electrochemical characterization}

$\mathrm{A} \mathrm{Ag} / \mathrm{AgCl}$ reference electrode was made by anode oxidization with $+0.5 \mathrm{~V}$ applied on the $\mathrm{Ag}$ filament in a $300 \mathrm{mM} \mathrm{KCl}$ solution for $30 \mathrm{~s}$. Electrodes were characterized by cyclic voltammetry $(\mathrm{CV})$ in the presence of the redox probe FcM. The concentration of FcM solution used in experiments was $2 \mathrm{mM}$ in $0.1 \mathrm{M}$ PBS. The scanning voltage

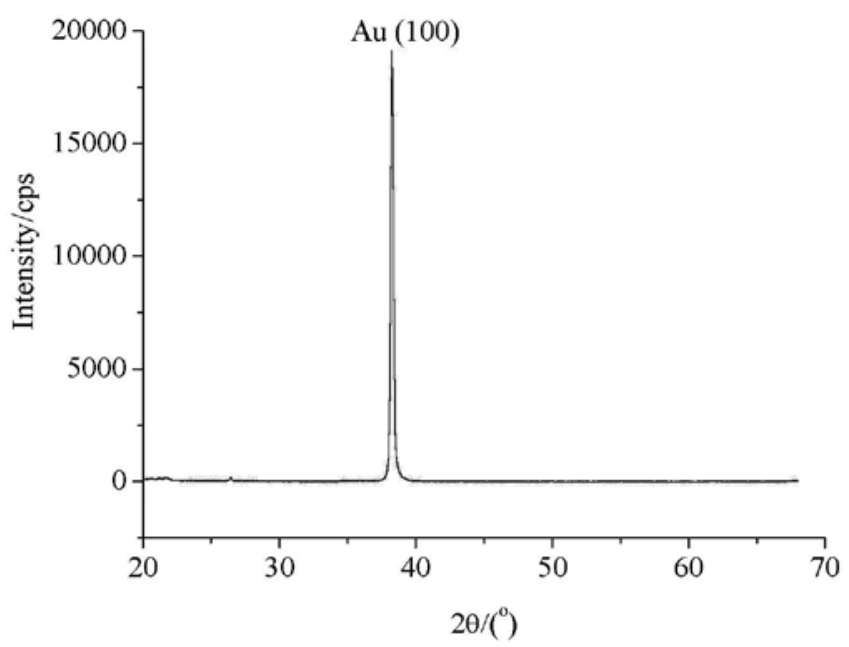

Fig. 1. XRD pattern of Au/Ti film. 
ranged from -0.5 to $0.5 \mathrm{~V}$ with a scanning rate of 50 or $100 \mathrm{mV} / \mathrm{s}$. CV was carried out using a CHI660C or CHI1030 workstation.

The compactness of the SAM on the Au electrode was characterized by CV. It is well known that $\mathrm{C}_{18} \mathrm{H}_{37} \mathrm{SH}$ can form a stable SAM on a Au substrate through the formation of a Au-S bond. The long carbon chains of a SAM can act similarly to an insulation layer to block ET between the bare Au electrode and redox species in the solution. In previous studies, there have been various methods to detect the compactness of the SAM, such as by AFM image analysis. Here we propose using CV to characterize the compactness of the SAM. CV was carried out after the Au electrode was soaked in $10 \mathrm{mM} \mathrm{C}_{18} \mathrm{H}_{37} \mathrm{SH}$ for different durations. The peak current indicates the compactness of the SAM.

To characterize the sensitivity of the electrochemical sensor modified by SGNFs, two key CV characteristics were analyzed. Firstly, the value of the peak current, which directly indicates strength of the sensing signal, was selected. Secondly, the Faradic current involved in $\mathrm{CV}$ was derived to determine the signal-to-noise ratio (SNR) of the sensor. The current in the CV curve is composed of Faradic and charge current. Only the Faradic current is an effective signal for electrochemical research, ${ }^{(1)}$ while the charge current is an interference signal. Theoretically, the Faradic current is proportional to the square root of the scan rate. Therefore, the SNR of the electrochemical system was characterized by the linearity of the relationship between the peak current and the square root of the scan rate.

\section{Results and Discussion}

\section{$3.1 \mathrm{n}$-Octadecyl mercaptan self-assembly on Au electrode surface}

Typical CV characteristics obtained in $0.10 \mathrm{M}$ PBS solution containing $2.0 \mathrm{mM} \mathrm{FcM}$ at the $\mathrm{Au}$ electrode prepared by immersion in $\mathrm{C}_{18} \mathrm{H}_{37} \mathrm{SH}$ ethanol solution for different durations are shown in Fig. 2. In Fig. 2, curve (a) is measured from a bare Au electrode (without $\mathrm{C}_{18} \mathrm{H}_{37} \mathrm{SH}$ on the electrode). The oxidation peak current is $2.8 \mu \mathrm{A}$. Curves (b) to (e) represent the measurements from $\mathrm{Au} / \mathrm{SAM}$ electrodes with SAM soaking durations of $3,6,24$, and $48 \mathrm{~h}$, respectively. Compared curve (b) to (a), it is clear that both the oxidation peak current and reduction peak current decrease a lot with an increasing peakto-peak potential. The absolute value of the oxidation peak decreases from 2.8 to $1.9 \mu \mathrm{A}$. There is still a large current, indicating that the SAM has not become sufficiently compact. Curves (c) and (d) show that with increasing immersion time, the peak current decreases. When the soaking time reaches $48 \mathrm{~h}$, the peak current decreases to about $0.5 \mu \mathrm{A}$ as shown in curve (e), which is an order of magnitude lower than that of the bare Au electrode. A highly compact SAM film was formed on the gold electrode surface. ET between the $\mathrm{Au}$ electrode and redox species was considerably blocked by the SAM of the long hydrocarbon chains.

\subsection{Controllable adsorption of SGNFs onto SAM/Au electrode}

The images obtained by scanning electron microscopy (SEM) and Raman spectra can be used to show the distribution and the structural differences between the SGNFs and graphite on the surface of the SAM/Au electrode.

The SEM images are shown in Fig. 3. Because of their hydrophobic property and high conjunction, SGNFs adsorbed onto the SAM appear as nano- and sub-microisolated 


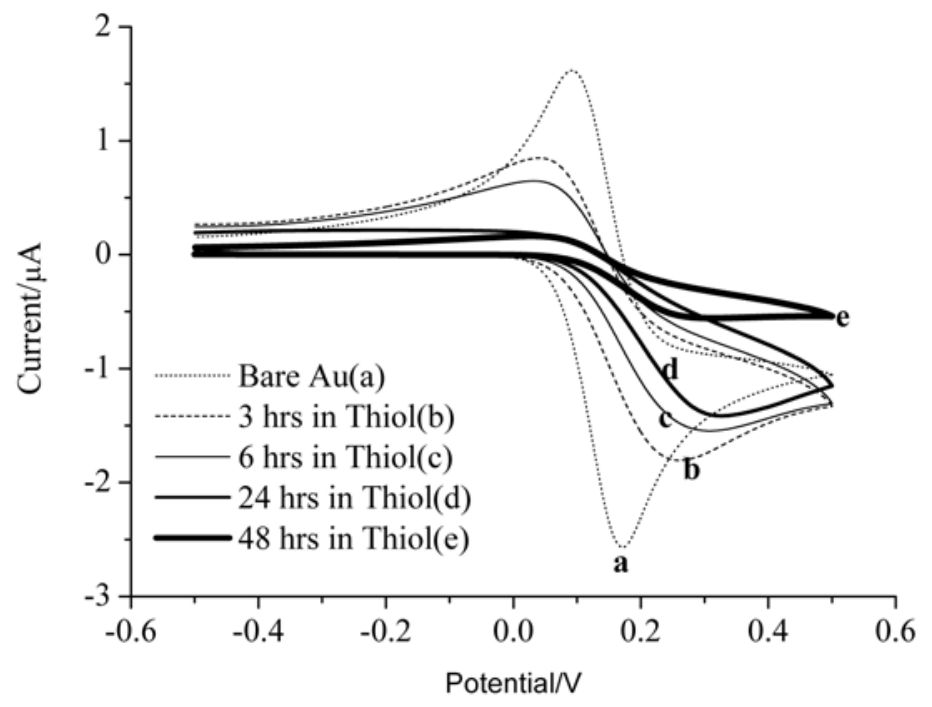

Fig. 2. $\mathrm{CV}$ curves of SAM/Au electrode soaked in $\mathrm{C}_{18} \mathrm{H}_{37} \mathrm{SH}$ solution for $0 \mathrm{~h}$ (curve a), $3 \mathrm{~h}$ (curve b), $6 \mathrm{~h}$ (curve c), $24 \mathrm{~h}$ (curve d), and $48 \mathrm{~h}$ (curve e), respectively. The scan rate of the CHI600C workstation was $50 \mathrm{mV} / \mathrm{s}$.
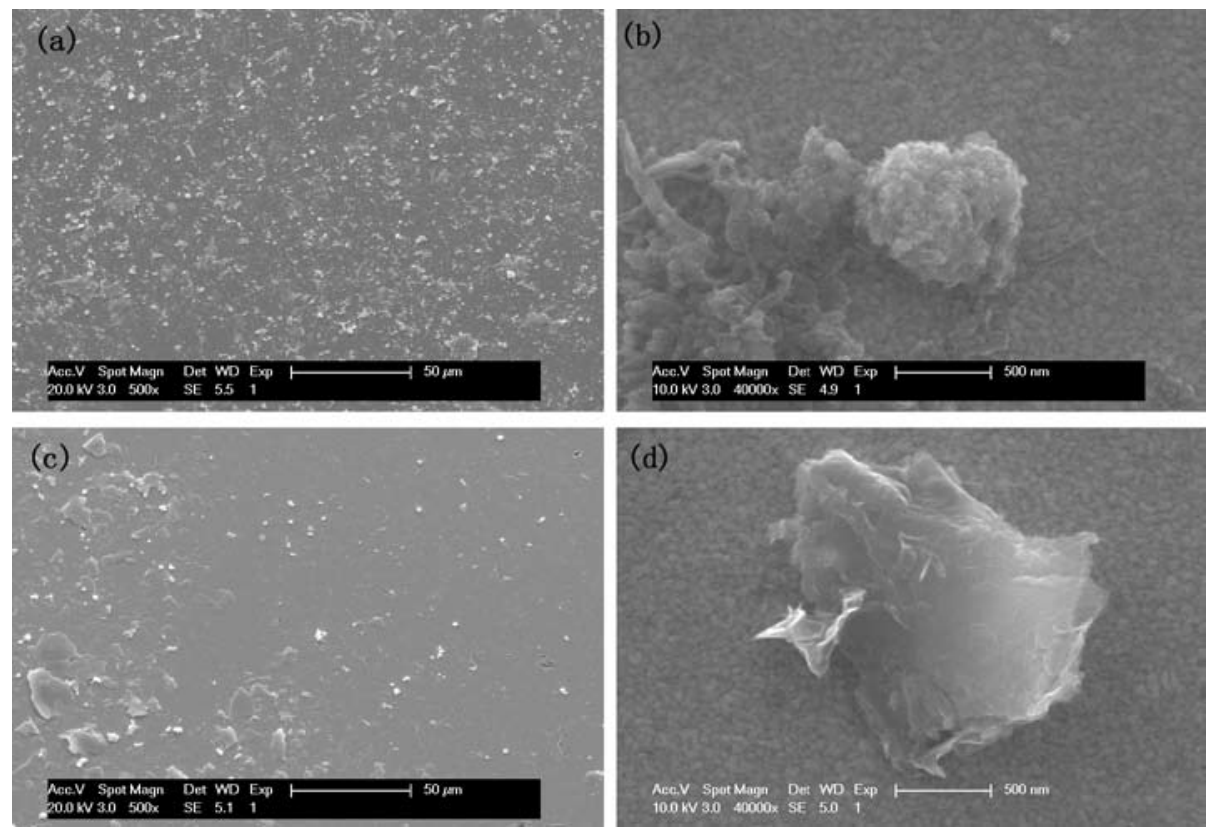

Fig. 3. SEM images of SGNF/SAM/Au electrode with magnification of (a) 500 times and (b) 40000 times; SEM images of graphite/SAM/Au electrode with magnification of (c) 500 times and (d) 40000 times. 
islands, rather than as a homogeneous monolayer, as shown in Fig. 3(a). The SEM image in Fig. 3(b) shows the structures of the SGNF islands in more detail, from which a high density of edge-plane SGNFs can be observed. Figures 3(c) and 3(d) are SEM images of the graphite/SAM/Au electrode. Comparing Figs. 3(b) and 3(d), we can clearly observe that SGNFs have a higher edge-plane density than graphite.

The above observation was also confirmed by the Raman spectra of the SGNF/ $\mathrm{SAM} / \mathrm{Au}$, graphite/SAM/Au and SAM/Au electrodes (see Fig. 4). From the spectra, it is clear that the SAM/Au electrode displays no obvious characteristic absorption peaks. However, the SGNF/SAM/Au electrode exhibits the prominent D band at $1325 \mathrm{~cm}^{-1}$, related to the $\mathrm{sp}^{3}$ form of carbon at the defects and ends of the sheets, and the $G$ band at $1579 \mathrm{~cm}^{-1}$, related to the $\mathrm{sp}^{2}$ form of the carbon. In the Raman spectrum of graphite/ $\mathrm{SAM} / \mathrm{Au}$, as expected, there exists a strong absorption peak ( $\mathrm{G}$ band) at $1579 \mathrm{~cm}^{-1}$ and a weak D band at $1334 \mathrm{~cm}^{-1}$, which suggests the regular structure of graphite. Comparing the $\mathrm{D} / \mathrm{G}$ peak ratio of $\mathrm{SGNF} / \mathrm{SAM} / \mathrm{Au}(\cong 2.31)$ with that of graphite/SAM/Au $(\cong 0.16)$, it can be concluded that the SGNFs exhibit a significantly higher number of edge/defect sites than the graphite does.

\subsection{Electrochemical characterization of $S G N F / S A M / A$ u electrode}

Figure 5 shows the $\mathrm{CV}$ curves of the different Au electrodes. Curves (a)-(d) are the $\mathrm{CV}$ curves of the bare $\mathrm{Au}$ electrode, the SAM/Au electrode, the graphite/SAM/Au electrode and the SGNF/SAM/Au electrode, respectively.

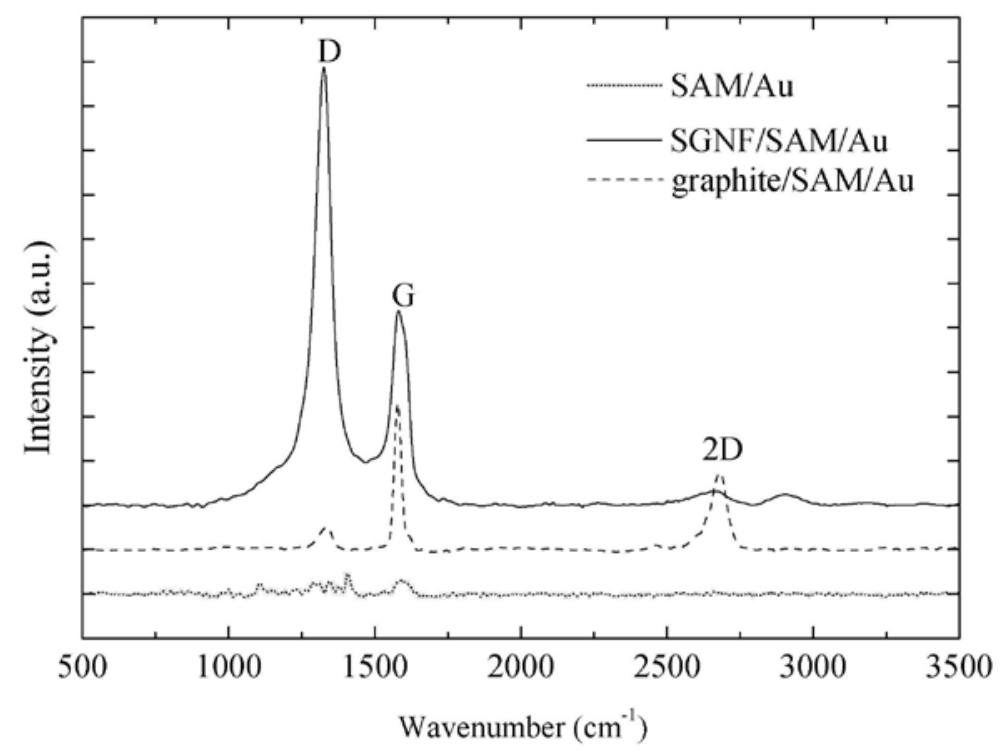

Fig. 4. Raman spectra of SGNF/SAM/Au (solid), graphite/SAM/Au (dashed), and SAM/Au (dotted) electrodes. All the spectra have been normalized. 


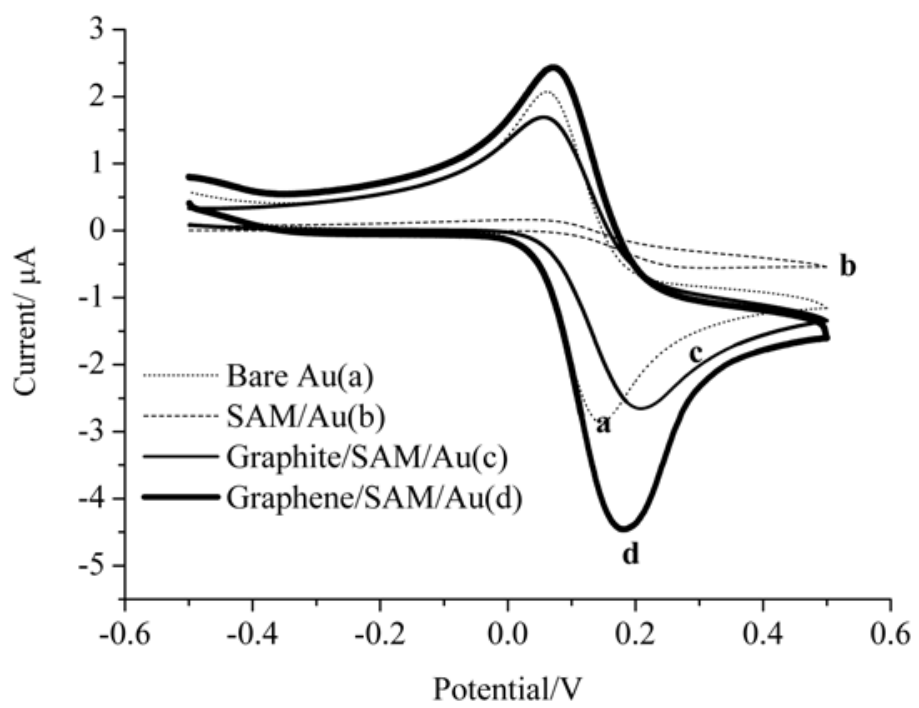

Fig. 5. CV curves of (a) Au electrode, (b) SAM/Au electrode, (c) graphite/SAM/Au, and (d) $\mathrm{SGNF} / \mathrm{SAM} / \mathrm{Au}$. The scan rate of the CHI1030 workstation was $0.1 \mathrm{~V} / \mathrm{s}$.

Curves (a) and (b) still show the occurrence of ET blocking by $\mathrm{C}_{18} \mathrm{H}_{37} \mathrm{SH}$ on the $\mathrm{SAM} / \mathrm{Au}$ electrode. Curve (c) shows the recovery of ET by the absorbed graphite particles on the graphite/SAM/Au electrode. The oxidation peak current reaches $2.8 \mu \mathrm{A}$ for the graphite/SAM/Au electrode, which is about $82.4 \%$ that of the $\mathrm{Au}$ electrode. Curve (d) shows the highest current peak. Such a high current implies that besides recovery, there still exists electron tunneling between the Au electrode and the electrolyte. The oxidation peak is $4.4 \mu \mathrm{A}$ for SGNFs, which is $33.5 \%$ higher than that of the bare Au electrode. SGNFs exhibit a faster observed heterogeneous electron transfer rate than graphite. This is why the peak current of the electrochemical reaction is larger for SGNF/SAM/Au than for graphite/SAM/Au.

The reason for the large response of the SGNF/SAM/Au electrode can be explained as follows. Although the surface of graphite particles is electrochemically active to some extent, it is far less active than that of graphene. Graphite cannot provide as many active electrons as SGNFs. SGNFs consist of a large number of graphene nanosheets with a base area of approximately $50 \times 50 \mathrm{~nm}^{2}$ and the fibers have a mean width of $40-50 \mathrm{~nm}$ and are 100-10000 nm long.(12) SGNFs contain an unprecedented number of open graphene edges on their surfaces (while having a graphene 'basal plane' surface only at the ends of the fibers). Since it is well established that the electrochemistry of carbon materials occurs on the 'edge plane' sites of graphene sheets while the 'basal-planes' are virtually electrochemically inactive, it is clear that we can expect much higher electrochemical activity from SGNFs than from other carbon materials. 
The scan rate plays a very important role in the study of electrochemical sensors and can be used to analyze the SNR of the electrochemical signal. CV was carried out with different scan rates on the bare Au electrode, the graphite/SAM/Au electrode, and the $\mathrm{SGNF} / \mathrm{SAM} / \mathrm{Au}$ electrode. Figure 6 shows the relationship between the oxidation peak current and scan rate. At a low scan rate, e.g., $0.05 \mathrm{v} / \mathrm{s}$, the $\mathrm{SGNF} / \mathrm{SAM} / \mathrm{Au}$ electrode has the largest oxidation peak current of $2.93 \mu \mathrm{A}$, whereas the graphite/SAM/Au electrode has the smallest oxidation peak current of $2.17 \mu \mathrm{A}$, and the oxidation peak current of the $\mathrm{Au}$ electrode is $2.41 \mu \mathrm{A}$. With the increase of the scan rate, the oxidation peak current of the graphite/SAM/Au electrode increases significantly slower than that of the bare electrode, and the oxidation peak current of the SGNF/SAM/Au electrode increases significantly faster than that of the bare electrode.

Figure 7 shows the relationship between the peak current and the square root of the scan rate for the gold electrode, the graphite/SAM/Au electrode, and the SGNF/SAM/Au electrode. The relationships can be fit linearly with correlation coefficients of 0.99946 , 0.99484, and 0.9537 for the $\mathrm{Au}, \mathrm{SGNF} / \mathrm{SAM} / \mathrm{Au}$, and graphite/SAM/Au electrodes, respectively.

The slope of the linear fitting line represents the effect of the Faradic current in the electrochemical signals, which determines the SNR. It can be seen that the SGNF/SAM/ $\mathrm{Au}$ electrode and the $\mathrm{Au}$ electrode have a higher SNR, whereas the graphite/SAM/Au electrode has a lower SNR. The slope of the fitting line of the SGNF/SAM/Au electrode is 10.638, which is larger than that of the bare electrode of 8.463. The larger slope of the $\mathrm{SGNF/SAM/Au} \mathrm{electrode} \mathrm{implies} \mathrm{its} \mathrm{higher} \mathrm{sensitivity} \mathrm{in} \mathrm{electrochemical} \mathrm{reactions} \mathrm{at} \mathrm{a}$ fast scan rate.

For the application of rapid detection, the SGNF/SAM/Au electrode can provide a high SNR induced by the high ET rate and sufficient number of active electrons.

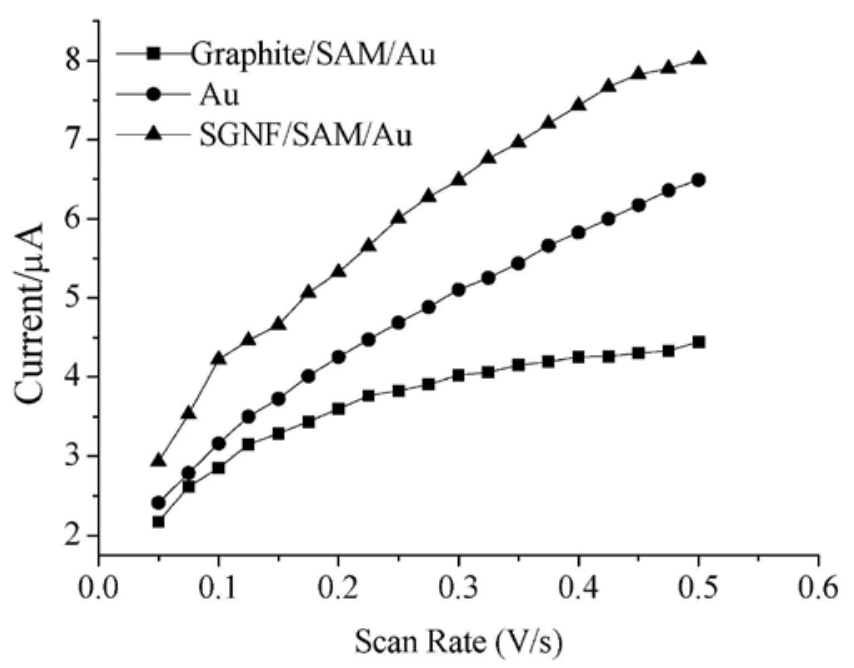

Fig. 6. Relationship between peak current and scan rate for $\mathrm{Au}$ electrode, graphite/SAM/Au electrode, and SGNF/SAM/Au electrode, measured by CHI1030 workstation. 


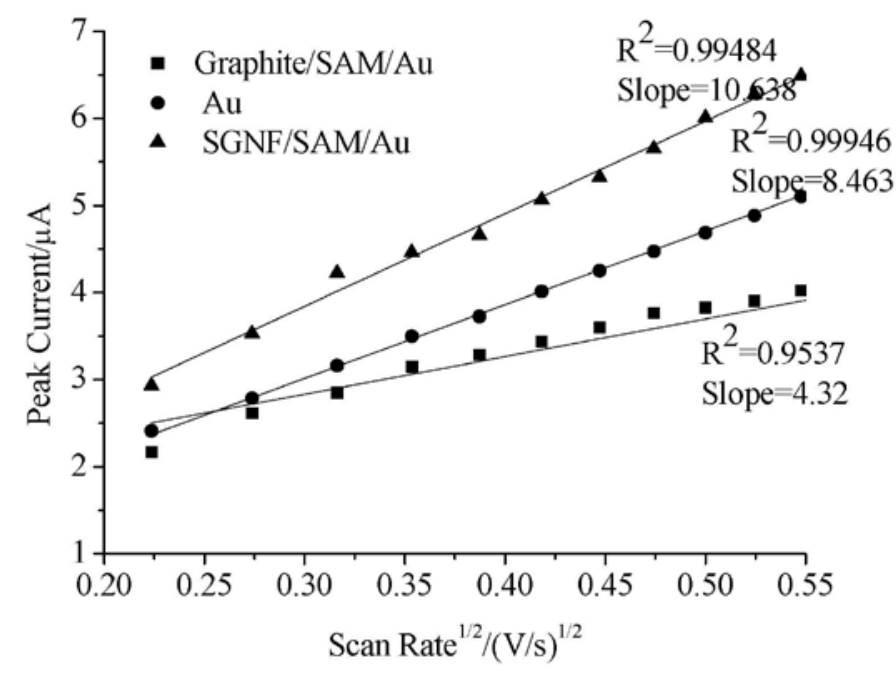

Fig. 7. Relationship between peak current and square root of scan rate for Au electrode, graphite/ $\mathrm{SAM} / \mathrm{Au}$ electrode and SGNF/SAM/Au electrode.

\section{Conclusions}

We have successfully fabricated an SGNF/SAM/Au electrode for electrochemical detection. SEM images and Raman spectra show that random nano- or sub-microarrays of graphene sheets can be formed onto the surface of a SAM/Au electrode. The adsorption of graphene sheets can recover heterogeneous ET between the gold electrode and redox species in an electrolyte. Electrochemical characterization shows that the SGNF/SAM/Au electrode has a higher peak current than the Au electrode and graphite/ $\mathrm{SAM} / \mathrm{Au}$ electrode, which implies a higher sensitivity by $\mathrm{CV}$ measurement. The $\mathrm{SGNF} / \mathrm{SAM} / \mathrm{Au}$ electrode demonstrates an excellent characteristic of Faradic current with a fitting slope of 10.638 and correlation coefficient of $R^{2}=0.9968$, implying that it can provide a high SNR in fast detection. The SGNF/SAM/Au electrode has potential application in rapid electrochemical detection with high sensitivity.

\section{Acknowledgements}

This work was supported by China's Megaprojects of Science Research for the 11th Five-Year Plan by Grant No. 2008ZX10003003 and the National Science Foundation of China by Grant Nos. 60876085 and 6176110.

The authors would like to thank Mr. Zhengxi Cheng of the Shanghai Institute of Technical Physics of the Chinese Academy of Sciences for his help in chip fabrication. 


\section{References}

1 K. S. Novoselov, A. K. Geim, S. V. Morozov, D. Jiang, Y. Zhang, S. V. Dubonos, A. A. Firsov and I. V. Grigorieva: Science 306 (2004) 666.

2 M. Pumera: Chem. Soc. Rev. 39 (2010) 4146.

3 C. L. Scott, G. Zhao and M. Pumera: Electrochem. Commun. 12 (2010) 1788.

4 A. Ambrosi, T. Sasaki and M. Pumera: Chem. Asian J. 5 (2010) 266.

5 A. Ambrosi and M. Pumera: Phys. Chem. Chem. Phys. 12 (2010) 8943.

6 H. O. Finklea, S. Avery and M. Lynch: Langmuir 3 (1987) 409.

7 D. Zheng, X. Li and J. Ye: Bioelectrochemistry 74 (2009) 240.

8 J. B. Shein, L. M. H. Lai, P. K. Eggers, M. N. Paddon-Row and J. J. Gooding: Langmuir 25 (2009) 11121.

9 A. Chou, P. K. Eggers, M. N. Paddon-Row and J. J. Gooding: J. Phys. Chem. C 113 (2009) 3203.

10 X. Xie, K. Zhao, X. Xu, W. Zhao, S. Liu, Z. Zhu, M. Li, Z. Shi and Y. Shao: J. Phys. Chem. C 114 (2010) 14243.

11 O. Ordeig, J. del Campo, F. X. Munoz, C. E. Banks and R. G. Compton: Electroanalysis 19 (2007) 1973.

12 http://www.strem.com/catalog/v/06-0170/12/carbon. 